\title{
PERIPHERAL CORNEAL OEDEMA FOLLOWING CATARACT EXTRACTION (BROWN-MCLEAN SYNDROME)
}

\author{
S. J. TUFT ${ }^{1}$, M. KERR MUIR ${ }^{2}$, E. S. SHERRARD ${ }^{1}$, R. J. BUCKLEY ${ }^{1}$ \\ London
}

\begin{abstract}
SUMMARY
We describe the clinical features of 21 eyes of 15 patients who developed peripheral bullous corneal oedema following cataract extraction. The peripheral oedema was asymptomatic in all cases and was only noted at a review examination. Although the onset of the peripheral oedema was probably delayed for several years after surgery, it was not documented to progress in any patient or to involve the central cornea. Endothelial specular microscopy did not reveal any endothelial features that are specific for this condition, but confirmed that the central endothelial cell density can be as high as $2150 \mathrm{cells} / \mathrm{mm}^{2}$ in the presence of peripheral corneal decompensation. Despite the surface irregularity resulting from the peripheral corneal oedema 12 patients ( 18 eyes) continued to wear contact lenses to correct their aphakia.
\end{abstract}

The delayed onset of peripheral bullous corneal oedema following cataract extraction was first described in 1969 by Brown and McLean, who reported the clinical features of eight patients who developed the condition a minimum of six years after intracapsular cataract surgery.' The Brown-McLean syndrome has been expanded in subsequent papers to include patients who develop oedema after extracapsular surgery, ocular injury, spontaneous lens resorption, or dislocation of the crystalline lens. ${ }^{2.3}$

The condition is characterised by bullous epithelial oedema and oedema of the underlying corneal stroma which extends for 1 to $3 \mathrm{~mm}$ from the limbus toward the central cornea. The oedema usually assumes a horseshoe configuration that spares the superior cornea adjacent to the cataract section. There is no associated corneal neovascularisation or anterior chamber inflammation, the anterior chamber angle is open, and discrete pigmented granules may be adherent to the endothelium beneath the oedematous areas. The oedema is typically only observed

Moorfields Eye Hospital, City Road, London EC1V 2PD, UK.

${ }^{2}$ St Thomas' Hospital, Lambeth Palace Road, London SE1 7EH, UK.

Correspondence to: Mr S. J. Tuft MD, FRACS, FCOphth, Moorfields Eye Hospital, City Road, London EC1V 2PD, UK. after a latent period of several years following surgery and the condition does not appear to progress, as extension of the oedema to involve the central cornea has not been reported. $^{1-3}$

Although the clinical features have been well described, the management and visual rehabilitation of patients with peripheral corneal oedema has received little attention. Patients with the Brown-McLean syndrome may have been wearing an aphakic contact lens correction prior to the development of the oedema, and they may wish to continue using contact lenses rather than to wear aphakic spectacles. However, it is known that the anoxic stress associated with contact lens wear can exacerbate corneal oedema, particularly if the endothelial cell functional reserve has been reduced by surgery. ${ }^{4}$ Thus patients with peripheral corneal oedema may be more susceptible to central corneal oedema during aphakic contact lens wear, and in addition, the irregular contour of the peripheral cornea could make comfortable lens fitting more difficult. We have therefore examined 21 aphakic eyes of 15 patients who developed peripheral bullous oedema following cataract surgery, 12 of whom continued to wear an aphakic contact lens correction. We report the clinical characteristics of these patients and our experience with contact lens fitting in this condition.

\section{PATIENTS AND METHODS}

Fifteen patients (21 aphakic eyes) who had developed peripheral corneal oedema following cataract extraction were referred for examination in the four year period 1988 to 1991. For each patient a record was made of the previous ocular history with particular attention to the details of surgery, the operative or post-operative complications, and the date when the corneal oedema was first documented. Details of the post-operative optical correction and the parameters of the patients' current contact lenses were also recorded. Clinical review included a slit lamp examination, applanation tonometry, gonioscopy, and an optical central corneal thickness measurement. The extent 
Table I. Clinical details of operated eyes

\begin{tabular}{|c|c|c|c|c|c|c|c|}
\hline Patient & & Operation & Associated risk factors & Latency (years) & Follow-up (years) & Acuity & Correction \\
\hline 1 & $58 \mathrm{M}$ & $\begin{array}{l}\text { Right ECCE* } \\
\text { Left ECCE* }\end{array}$ & Hypopyon uveitis & $\begin{array}{l}12 \\
\text { Band keratopathy }\end{array}$ & 2 & $6 / 6$ & Glasses \\
\hline 2 & $61 \mathrm{M}$ & Right ICCE & Iris prolapse & 23 & 2 & $6 / 5$ & DWRCL† \\
\hline \multirow[t]{2}{*}{3} & $45 \mathrm{~F}$ & Right ICCE & Retinal detachment & 5 & 6 & $6 / 9$ & DWRCL \\
\hline & & Left ICCE & Nil & 4 & 5 & $6 / 6$ & DWRCL \\
\hline 4 & $70 \mathrm{M}$ & Right ICCE & Nil & 17.5 & 2 & $6 / 12$ & DWRCL \\
\hline \multirow[t]{2}{*}{5} & $68 \mathrm{~F}$ & Right ICCE & High myopia & 10 & 4 & $6 / 9$ & DWRCL \\
\hline & & Left ICCE & High myopia & 7 & 1.5 & $6 / 18$ & DWRCL \\
\hline \multirow[t]{2}{*}{6} & $64 \mathrm{M}$ & Right ICCE & Nil & Bullous keratopathy & & NPL & \\
\hline & & Left ICCE & Nil & 13 & 6 & $6 / 9$ & DWRCL \\
\hline \multirow[t]{2}{*}{7} & $81 \mathrm{~F}$ & Right ICCE & Nucleus dislocated, vitrectomy & 6 & 2 & $6 / 9$ & EWSCL \\
\hline & & Left ICCE & Nil & 5 & 2 & $6 / 6$ & EWSCL \\
\hline \multirow[t]{2}{*}{8} & $77 \mathrm{~F}$ & Right ICCE & Nil & 23 & 3 & $6 / 6$ & DWRCL \\
\hline & & Left ICCE & & 13 & 3 & $6 / 6$ & DWRCL \\
\hline 9 & $47 \mathrm{M}$ & Right ICCE & Vitreous loss & 18 & 6 & $6 / 9$ & Nil (diplopia) \\
\hline \multirow[t]{2}{*}{10} & $66 \mathrm{~F}$ & Right ECCE & Chronic open angle glaucoma & 33 & 2 & $6 / 6$ & Scleral \\
\hline & & Left ECCE & & 33 & 2 & $6 / 6$ & Scleral \\
\hline 11 & $80 \mathrm{~F}$ & Left ICCE & High myopia & 21 & 22 & $6 / 9$ & Scleral \\
\hline 12 & $70 \mathrm{~F}$ & Right ICCE & Nil & 1 & 2 & $6 / 5$ & DWRCL \\
\hline \multirow[t]{2}{*}{13} & $32 \mathrm{~F}$ & Right ICCE & & Normal cornea & & & \\
\hline & & Left ICCE & Vitrectomy for endophthalmitis & 7 & 2 & $6 / 9$ & Glasses \\
\hline \multirow{2}{*}{14} & $50 \mathrm{M}$ & Right ECCE* & & 21 & 17 & $6 / 12$ & DWRCL \\
\hline & & Left ECCE* & Retinal detachments & Normal cornea & & NPL & \\
\hline \multirow[t]{2}{*}{15} & $71 \mathrm{M}$ & Right ICCE & Retinal detachment & 5 & 8 & $6 / 6$ & DWRCL \\
\hline & & Left ICCE & Retinal detachment & 6 & 8 & $6 / 6$ & DWRCL \\
\hline
\end{tabular}

*Needling and washout, posterior capsule appeared intact.

tHad secondary anterior chamber lens implantation.

DWRCL daily wear rigid contact lens, EWSCL extended wear soft contact lens, ICCE intracapsular extraction, ECCE extracapsular cataract extraction. Latency = interval following last surgical procedure.

of the corneal oedema was recorded diagrammatically. Clinical photographs were taken of abnormal features and nine of the patients consented to undergo wide field specular microscopy (Keeler-Konan).

\section{RESULTS}

There were seven male and eight female patients with an age range from 32 to 81 years (mean 63 years) at the time of observation (Table I). In 16 eyes the oedema spared the superior quadrant of the cornea (Fig. 1), while in five eyes the entire circumference of the peripheral cornea was involved. The five eyes with circumferential oedema

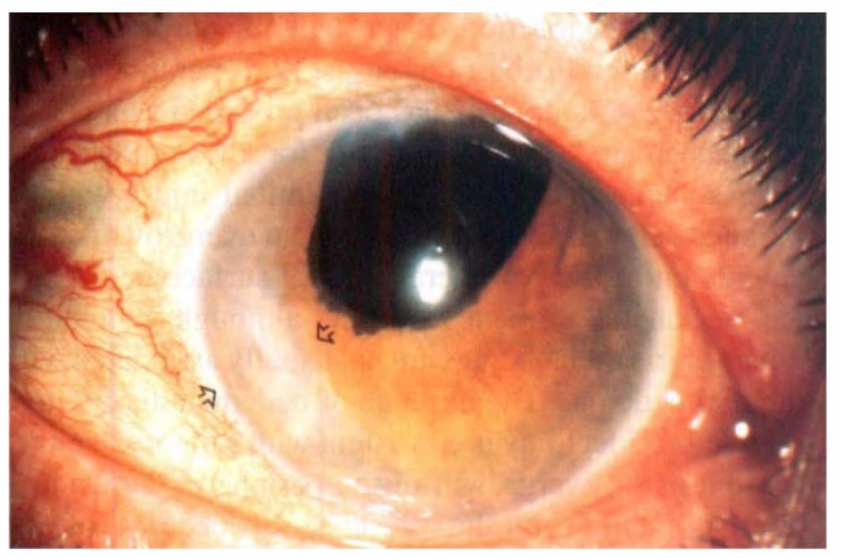

Fig. 1. Clinical photograph of peripheral corneal oedema predominantly affecting the infero-temporal cornea (arrows) of a patient following an intracapsular extraction and a broad sector iridectomy. Although there is stromal opacity in the superior cornea adjacent to the cataract section this is not associated with epithelial oedema. either had small peripheral iridectomies or iris incarceration to the posterior surface of the cataract section, while the eyes with sectorial oedema all had broad iridectomies. There was no peripheral corneal vascularisation, peripheral iris atrophy, or pseudo-exfoliation in any eye. On slit lamp examination there appeared to be a line of demarcation on the endothelium that corresponded to the forward edge of the overlying oedema, and orange-brown pigmented granules were most numerous along this line. The intraocular pressure was less than $20 \mathrm{mmHg}$ in all eyes and all affected eyes had widely open anterior chamber angles in areas away from the cataract section. The central corneal thickness was less than $0.54 \mathrm{~mm}$ in all but one eye $(0.58 \mathrm{~mm})$.

Seventeen of the affected eyes had undergone intracapsular cataract extraction (ICCE), two had had an extracapsular cataract extraction (ECCE) and two had aspiration of lens remnants with preservation of part of the posterior capsule following needling of congenital cataracts. No patient had an intraocular lens at the time of examination. Two patients were known to be highly myopic ( $>8$ dioptres) prior to surgery and one had received retinal xenon photocoagulation of peripheral retinal breaks prior to cataract extraction. One patient had a history of recurrent anterior uveitis prior to surgery and one patient was receiving treatment for chronic open angle glaucoma; no other risk factors for surgical endothelial cell loss were identified in the remaining patients.

Although operative complications (dislocated lens nucleus, vitreous loss) and post-operative complications (hypopyon, iris prolapse, or retinal detachment) were recorded in six patients, no patient was recorded to have 
had a flat anterior chamber in the post-operative period. Patient \# 13 has Marfan's syndrome and bilateral ICCEs were performed for superiorly dislocated lenses; this patient had a leaking bleb in one eye and developed peripheral corneal oedema and secondary glaucoma following a therapeutic vitrectomy for endophthalmitis. Patient \#6 developed aphakic bullous keratopathy and absolute glaucoma in his contralateral eye soon after a traumatic cataract extraction, but an intervening period of oedema limited to the peripheral cornea was not documented.

The mean latency between the last surgical event and the documentation of peripheral corneal oedema was 13.5 years (range 1 to 33 years), and the mean follow-up subsequent to the documentation of oedema was 5.1 years (range 1.5 to 22 years). In no patients has there been an extension of the peripheral oedema to involve the central cornea. In every patient the recognition of peripheral oedema appears to have been a coincidental observation during routine review; we could obtain no definite history of an onset of discomfort, loss of contact lens tolerance or foreign-body sensation corresponding to the onset of peripheral oedema. However, as these patients had not been under continuous review following their surgery and as they were asymptomatic, we recognise that a considerable delay may have occurred between the development and the recognition of the condition.

At the time of examination 18 eyes ( 12 patients) were wearing contact lenses. Twelve eyes had been fitted with gas permeable daily wear rigid contact lenses, one elderly patient was unable to cope with daily wear lenses and had been fitted with bilateral extended wear soft contact lenses elsewhere, and two patients wore PMMA scleral contact lenses. No patient had experienced significant contact lens associated disease, such as microbial keratitis. Three patients were not wearing contact lenses; Patient \#1 had always worn aphakic spectacles, and patients \#9 and \# 13 had abandoned contact lens wear before oedema was noted. Patient \#2 was unhappy with unilateral contact lens wear and had a secondary anterior chamber intraocular lens implanted at the referring hospital.

Specular photographs from the central corneal endothelium were obtained from nine patients, and these demonstrated endothelial cell polymegethism and a cell density (mean 1850, range 1190 to 2150 cells $/ \mathrm{mm}^{2}$ ) that was lower than is normal for this age group, but which was consistent with previous intracapsular surgery. Scattered central cornea guttata were observed in two patients. As stromal oedema prevented visualisation of the peripheral endothelium we can not comment on the morphology of the endothelial cells beneath the oedematous areas, but there was no evidence of an annular dystrophy of the peripheral endothelium in any of the five unoperated fellow eyes in this series.

\section{DISCUSSION}

We have confirmed previous observations of the clinical features of the Brown-McLean syndrome, in particular the apparent delay between the surgery and the appearance of corneal oedema. Although the oedema usually spares the superior cornea, in five eyes where there was no patent iridectomy or only a small peripheral iridectomy the oedema formed a complete ring. We noted pigment deposition on the endothelium beneath the peripheral oedema, but consider that this sign is not specific for this syndrome as we have observed a similar pattern of pigment dispersion in eyes following ICCE in which there is no sign of peripheral corneal decompensation. The appearance of the central endothelium in these patients has been reported to be normal, ' but using endothelial specular microscopy we observed a reduced endothelial cell density on the central cornea with polymegethism. However, these changes are not specific and are compatible with previous intraocular surgery. ${ }^{5}$

The minimum latency for the recognition of the peripheral oedema in our patients was one year, but a latency of up to 34 years has been reported. .' However, any figures for latency can only be an approximation as patients are usually asymptomatic and not regularly reviewed, thus the identification of the onset of this condition may be delayed. We have no evidence for the central progression of the peripheral oedema to involve the entire cornea, but over a period of years one would expect a small proportion of eyes that had undergone traumatic intraocular surgery to develop central corneal decompensation as a result of endothelial cell loss. One patient in this series had total bullous oedema of his contralateral eye at the time of examination, but he had not been documented to pass through an intermediate period of oedema limited to the peripheral cornea.

The aetiology of this condition is uncertain, as there does not seem to be any association with a pre-existing endothelial dystrophy of the peripheral cornea. Trauma to the peripheral endothelium at the time of surgery may play a role as operative complications or post-operative inflammation occurred in at least five eyes $(24 \%)$ in this series, and $38 \%$ of the series of Charlin had signs of surgical complications. ${ }^{3}$ Other possible predisposing factors such as high myopia, dislocation of the lens, or chronic uveitis occurred in a minority of our cases. Postoperative microtrauma from irododonesis has been proposed as a causative mechanism, ' but the failure for the condition to progress suggests a localised and limited injury without continued cell loss. It is possible that an overgrowth of iris elements on to the peripheral corneal surface could produce peripheral corneal oedema, although pigmented plaques of iris melanocytes are not usually associated with epithelial oedema. ${ }^{7}$ The absence of oedema adjacent to a large sector iridectomy is consistent with a theory of either iridodonesis or overgrowth of the peripheral cornea as a causative factor, but the presence of oedema of the superior cornea in some patients argues against protection by the upper lid determining the sparing of the upper cornea. Anoxia or injury to the peripheral cornea caused by contact lens wear does not seem to play a role as some patients in this series, and in previous series, had never worn contact lenses. 
Peripheral corneal oedema may develop in other situations. A differential diagnosis includes the iridocorneal endothelial syndrome, although this is typically unilateral with extensive peripheral anterior synechiae associated with areas of oedema and a characteristic endothelial cell morphology. Peripheral oedema may also develop as a result of traumatic disinsertion of Descemet's membrane, but this is usually localised to an area adjacent to a cataract section. We have also observed transient peripheral oedema in presumed herpetic keratouveitis. A ring of pigment deposition on the corneal side of Schwalbe's line, a clinical sign in the Brown-McLean syndrome, also occurs in the majority of patients with pseudo-exfoliation of the lens capsule. ${ }^{8}$

We do not consider that the development of peripheral corneal oedema is an absolute contraindication to continued contact lens wear and a number of different lens designs are tolerated. Twelve patients ( 18 eyes) have worn contact lenses for periods of up to 22 years following the recognition of their peripheral corneal oedema. Two patients who were already wearing PMMA scleral contact lenses have continued with these lenses, and this type of lens offers the opportunity to bridge the peripheral corneal irregularity if this is thought desirable. The fact that a proportion of the patients wore the same lens type before and after the onset of oedema suggests that the oedema has a minor effect on contact lens tolerance. Although there is a high incidence of microbial keratitis in patients who wear a therapeutic contact lens for bullous keratopathy," the small numbers in this series preclude comment on whether there is an increased risk of wearing a contact lens in the presence of peripheral corneal oedema.

We have no evidence that the central corneas of these patients are particularly susceptible to hypoxia but we have replaced lenses with a high Dk rigid gas-permeable lens material with good wetting characteristics wherever possible, and have suggested that the daily duration of contact lens wear is kept to a minimum. We have tried to limit mechanical trauma to the peripheral cornea by using an interpalpebral fit and have increased the diameter of the lens in patients who have tended to develop 3 and 9 o'clock staining on areas of oedema. A continuous curve (aspheric) lens with a well-blended curve at the margin is chosen to reduce indentation of the lens edge at the corneal periphery. We consider that secondary intraocular lens implantation should only be considered for patients who become intolerant to contact lens wear.

The authors thank Mr P. J. Holmes-Sellors, Mr M. O’Riordan, Mr J. K. G. Dart, and Dr Lali Moodaley for permission to report on their patients.

Key words: Cataract. cornea, corneal oedema.

\section{REFERENCES}

1. Brown SI, McLean JM: Peripheral corneal edema after cataract extraction. A new clinical entity. Trans Am Acad Ophthalmol Otolaryngol 1969, 73: 465-9.

2. Brown SI: Peripheral corneal edema after cataract extraction. Am J Ophthalmol 1970, 70: 326-8.

3. Charlín R: Peripheral corneal edema after cataract extraction. Am J Ophthalmol 1985, 99: 298-303.

4. Mandell RB, Polse KA, Fatt I: Corneal swelling caused by contact lens wear. Arch Ophthalmol 1970, 83: 3-9.

5. Matsuda M, Suda T, Manabe R: Serial alterations in endothelial cell shape and pattern after intraocular surgery. Am J Ophthalmol 1984, 98: 313-9.

6. Lim JI, Lam S, Sugar J: Brown-McLean syndrome. Arch Ophthalmol 1991, 109: 21-2.

7. Kampik A, Patrinely JR, Green WR: Morphologic and clinical features of retrocorneal melanin pigmentation and pigmented pupillary membranes: a review of 225 cases. Surv, Ophthalmol 1982, 27: 161-80.

8. Duke-Elder S: System of Ophthalmology. Volume XI; Disorders of the Lens and Vitreous. London. Henry Kimpton, 1969: 51.

9. Graham C.M. Dart JKG, Buckley RJ: Extended wear hydrogel end daily wear hard contact lenses for aphakia. Ophthalmology 1986, 93: 1489-94. 